Pacific Journal of Mathematic 


\section{ON A TAUBERIAN THEOREM FOR ABEL SUMMABILITY}

\section{Otто SzÁsZ}

1. Introduction. In 1928 the author proved the following theorem [2, Section 2]:

THEOREM A. If $p>1$ and

$$
\sum_{\nu=1}^{n} \nu^{p}\left|a_{\nu}\right|^{p}=O(n), \quad n \rightarrow \infty,
$$

then Abel summability of the series $\sum_{n=0}^{\infty} a_{n}$ to $s$ implies its convergence to $s$.

The theorem is the more general the smaller $p$ is; it does not hold for $p=1$ [2, Section 1; 1, pp.119,122]. However, for this case Rényi proved the following theorem:

THEOREM B. If

$$
\lim _{n \rightarrow \infty} \frac{1}{n} \sum_{\nu=1}^{n} \nu\left|a_{\nu}\right|=\iota<\infty
$$

exists, then Abel summability of $\sum_{n=0}^{\infty} a_{n}$ to $s$ implies convergence of the series to $s$.

2. Generalization. We give a simpler proof and at the same time a slight generalization of The orem B.

The OREм 1. Assume that

$$
V_{n}=\sum_{\nu=1}^{n} \nu\left|a_{\nu}\right|=O(n),
$$

and that

$$
\frac{1}{m} V_{m}-\frac{1}{n} V_{n} \rightarrow 0
$$

Received April 10, 1950. The preparation of this paper was sponsored (in part) by the Office of Naval Research.

Pacific J. Nath. I (1951), 117-125. 
for every sequence $m=m_{n}$, such that $m_{n} / n \longrightarrow 1$ as $n \longrightarrow \infty$. Then Abel summa bility to $s$ of $\sum_{n=0}^{\infty} a_{n}$ implies its convergence to $s$.

Property (2.2) is called slow oscillation of the sequence $V_{n} / n$.

Proof of Theorem 1 . We write

$$
\sum_{\nu=0}^{n} a_{\nu}=s_{n}, \quad \sum_{\nu=0}^{n} s_{\nu}=(n+1) \sigma_{n} .
$$

It is easy to verify that, for $k=0,1,2, \cdots$, we have

(2.3) $s_{n-1}-\sigma_{n+k}=\frac{n}{k+1}\left(\sigma_{n+k}-\sigma_{n-1}\right)-\frac{1}{k+1} \sum_{\nu=0}^{k}(k+1-\nu) a_{n+\nu}$.

It is known [see 2, Section 2] that if for a finite $s$ we have

$$
\lim _{x \rightarrow 1} \sum_{n=0}^{\infty} a_{n} x^{n}=s,
$$

then (2.1) implies $\sigma_{n} \longrightarrow s$; thus, if

$$
\operatorname{liu.b}_{k \geq 0} \cdot\left|\sigma_{n-1}-\sigma_{n+k}\right|=\epsilon_{n} \text {, }
$$

then $\epsilon_{n} \longrightarrow 0$.

We now choose

$$
k=k_{n}=\left[n \epsilon_{n}^{1 / 2}\right], \quad \text { so that } \quad k \leq n \epsilon_{n}^{1 / 2}<k+1 \text {; }
$$

it follows, in view of (2.4), that

$$
\frac{n}{k+1}\left|\sigma_{n-1}-\sigma_{n+k}\right|<\epsilon_{n}^{1 / 2}
$$

In view of (2.3) our theorem will be proved if we show that

$$
\frac{1}{k+1} \sum_{\nu=0}^{k}(k+1-\nu) a_{n+\nu} \longrightarrow 0 \text {, }
$$


Now

$$
\begin{aligned}
\frac{1}{k+1} \mid \sum_{\nu=0}^{k}(k & +1-\nu) a_{n+\nu} \mid \\
& \leq \frac{1}{k+1} \sum_{\nu=0}^{k}(n+\nu)\left|a_{n+\nu}\right| \frac{k+1-\nu}{n+\nu} \leq \frac{1}{n}\left(V_{n+k}-V_{n-1}\right),
\end{aligned}
$$

and

$$
\begin{aligned}
\frac{1}{n}\left(V_{n+k}-V_{n-1}\right)= & \frac{V_{n+k}}{n+k} \cdot \frac{n+k}{n}-\frac{V_{n-1}}{n-1} \cdot \frac{n-1}{n} \\
= & \frac{V_{n+k}}{n+k}-\frac{V_{n-1}}{n-1}+\frac{k}{n} \frac{V_{n+k}}{n+k}+\frac{1}{n} \frac{V_{n-1}}{n-1} ;
\end{aligned}
$$

using (2.2) and (2.5), we see that

$$
\frac{1}{n}\left(V_{n+k}-V_{n-1}\right) \rightarrow 0 \quad \text { as } \frac{k}{n} \rightarrow 0 \quad \text { and } n \rightarrow \infty \text {, }
$$

and thus Theorem 1 is proved.

Rényi observed that the Theorems $\mathrm{A}$ and $\mathrm{B}$ are overlapping. We now show that Theorem 1 includes not only Theorem B, but also Theorem A. Clearly (2.1) follows from (1.1) by Hölder's inequality. Furthermore,

$$
\begin{aligned}
V_{n+k}-V_{n}=\sum_{\nu=n+1}^{n+k} \nu\left|a_{\nu}\right| \leq k^{(p-1) / p}\left(\sum_{\nu=n+1}^{n+k} \nu^{p}\left|a_{\nu}\right|^{p}\right)^{1 / p} & \\
& =k^{(p-1) / p} O\left[(n+k)^{1 / p}\right] ;
\end{aligned}
$$

hence,

$$
\frac{1}{n}\left(V_{n+k}-V_{n}\right)=\frac{k}{n} O\left[\left(\frac{n}{k}\right)^{1 / p} \cdot\right]=O\left[\left(\frac{k}{n}\right)^{(p-1) / p}\right] \rightarrow 0 \quad \text { as } \frac{k}{n} \rightarrow 0 .
$$

It now follows from (2.6) that (2.2) holds; thus (1.1) implies (2.1) and (2.2), which proves our assertion.

An example of a sequence $V_{n}>0$, and increasing, for which (2.2) holds, 
while $n^{-1} V_{n} \uparrow \infty$, is

$$
V_{n}=n \log n, \quad n \geq 2,
$$

because

$$
\frac{V_{n+k}}{n+k}-\frac{V_{n}}{n}=\log \left(1+\frac{k}{n}\right) \rightarrow 0, \quad \text { as } \frac{k}{n} \longrightarrow 0, \quad n \rightarrow \infty .
$$

3. A more general result. A generalization of Theorem A is the following [see $5, \mathrm{p} .56]$ :

THEOREM A'. If for some $p>1$, we have

$$
\sum_{\nu=1}^{n} \nu^{p}\left(\left|a_{\nu}\right|-a_{\nu}\right)^{p}=O(n)
$$

then the Abel summability of $\Sigma_{n=0}^{\infty} a_{n}$ implies its convergence to the same value.

An analogue to Theorem 1 is the theorem:

Theorem 2. Assume that

$$
U_{n}=\sum_{\nu=1}^{n} \nu\left(\left|a_{\nu}\right|-a_{\nu}\right)=O(n)
$$

and that

$$
\frac{1}{m} U_{m}-\frac{1}{n} U_{n} \rightarrow 0
$$$$
\text { as } \frac{m}{n} \longrightarrow 1, \quad n \rightarrow \infty
$$

If now $\sum_{n=0}^{\infty} a_{n}$ is Abel summable to $s$, then it converges to $s$.

Proof of Theorem 2. We have

$$
-\sum_{\nu=1}^{n} \nu a_{\nu} \leq \sum_{\nu=1}^{n} \nu\left(\left|a_{\nu}\right|-a_{\nu}\right)=O(n)
$$

hence [see 5, the Lemma on p.52] Abel summability of $\sum_{n=0}^{\infty} a_{n}$ implies its summability $(C, 1)$. From $(2.3)$ we have 


$$
\begin{aligned}
s_{n-1}-\sigma_{n+k} & \leq \frac{n}{k+1}\left(\sigma_{n+k}-\sigma_{n-1}\right) \\
& +\frac{1}{k+1} \sum_{\nu=0}^{k}(k+1-\nu)\left(\left|a_{n+\nu}\right|-a_{n+\nu}\right)
\end{aligned}
$$

from (2.4) anà (2.5) we obtain

$$
\frac{n}{k+1}\left(\sigma_{n+k}-\sigma_{n-1}\right)<\epsilon_{n}^{1 / 2}
$$

Using the same argument as in the proof of Theorem 1 , replacing $V_{n}$ by $U_{n}$, we find that

$$
\lim _{n \rightarrow \infty} \sup _{n} \leq s
$$

We next employ the identity, similar to (2.3),

$$
\begin{aligned}
& s_{n}-\sigma_{n-k-1}=\frac{n+1}{k+1}\left(\sigma_{n}-\sigma_{n-k-1}\right) \\
& \quad+\frac{1}{k+1} \sum_{\nu=0}^{k}(k-\nu) a_{n-\nu}, \quad k=0,1,2, \cdots,
\end{aligned}
$$

and the inequality

$$
a_{\nu} \geq a_{\nu}-\left|a_{\nu}\right|
$$

The same reasoning as before now yields

$$
\lim _{n \rightarrow \infty} \inf s_{n} \geq s
$$

Finally (3.4) and (3.5) prove Theorem 2.

It is clear from the proof that condition (3.3) can be replaced by

$$
\frac{1}{n}\left(U_{m}-U_{n}\right) \rightarrow 0, \quad \text { as } \frac{m}{n} \rightarrow 1, \quad n \rightarrow \infty \text {. }
$$

4. An equivalent result. A glance at the proof of Theorem 1 shows that the following lemma holds: 
LEMMA 1. If $V_{n}$ is positive and monotone increasing, and if

$$
V_{n}=O(n)
$$

as $n \longrightarrow \infty$,

and (2.2) holds, then

$$
\frac{1}{n}\left(V_{m}-V_{n}\right) \rightarrow 0
$$

as $\frac{m}{n} \rightarrow 1$,

$n \rightarrow \infty$

We now prove the inverse:

LEMMA 2. If $V_{n}>0$, and increasing, and if (4.2) holds, then (4.1) and (2.2) hold.

Proof. We write

$$
V_{n}=n \omega_{n}, \quad \omega_{n} \geq 0,
$$

and

$$
\frac{1}{n}\left(V_{m}-V_{n}\right)=\omega_{m}-\omega_{n}+\left(\frac{m}{n}-1\right) \omega_{m} .
$$

Let

$$
\max _{\nu \leq n} \omega_{\nu}=\rho_{n} ;
$$

then $\rho_{n} \uparrow \rho \leq \infty$. If $\rho<\infty$, then $V_{n}=O(n)$. Suppose now that $\rho=\infty$; then there are infinitely many indices $m=m_{\nu}$, so that $\omega_{m}=\rho_{m}$ for $m=m_{\nu}, \nu=1,2,3, \cdots$. For these $m$ and for $n<m$, from (4.3) we get

$$
\frac{1}{n}\left(V_{m}-V_{n}\right)>\left(\frac{m}{n}-1\right) \rho_{m} .
$$

We now choose

$$
n=\frac{m \rho_{m}{ }^{1 / 2}}{1+\rho_{m}{ }^{1 / 2}}<m
$$

so that

$$
\frac{m}{n}=\frac{1+\rho_{m}{ }^{1 / 2}}{\rho_{m}{ }^{1 / 2}} \rightarrow 1 ;
$$


then, using (4.4), we have

$$
\frac{1}{n}\left(V_{m}-V_{n}\right)>\rho_{m}^{1 / 2} \rightarrow \infty
$$

in contradiction to the assumption (4.2). It follows that (4.1) holds; finally (2.2) follows from (4.1), (4.2), and (4.3). This proves Lemma 2.

We now prove the following theorem:

THEOREM 3. Let $U_{n}=\sum_{\nu=1}^{n} \nu\left(\left|a_{\nu}\right|-a_{\nu}\right)$; if

$$
\frac{1}{n}\left(U_{m}-U_{n}\right) \rightarrow 0, \quad \text { as } \frac{m}{n} \rightarrow 1, \quad n \rightarrow \infty,
$$

and if $\sum_{n=0}^{\infty} a_{n}$ is Abel summable, then $\sum_{n=0}^{\infty} a_{n}$ is convergent to the same value.

Proof of Theorem 3. In view of Lemma 2, Theorem 3 includes Theorem 2; it also includes Theorem 1 , because of Lemma 2, and of the inequality

$$
U_{m}-U_{n} \leq 2\left(V_{m}-V_{n}\right) \text {, }
$$$$
m>n \text {. }
$$

Conversely, by Lemma 2, (4.5) implies (3.2) and (3.3), so that Theorem 3 is equivalent to Theorem 2 , and is thus valid.

To show that Theorem 1 is actually more general than Theorem $B$ we give an example of a sequence $\omega_{n}$ so that $n \omega_{n}$ is increasing, $\omega_{n}$ is slowly oscillating and $\omega_{n}=O(1)$, but $\lim \omega_{n}$ does not exist. Let

$$
\omega_{n}=\sum_{\nu=1}^{n} \nu^{-1} \epsilon_{\nu}, \quad \text { where } \epsilon_{\nu}= \pm 1
$$

choose $\epsilon_{\nu}=+1$ as long as $\omega_{n} \leq 3 ; \nu=1,2, \cdots, n_{1}$, say. Choose $\epsilon_{\nu}=-1$ as long as $\omega_{n} \geq 2 ; \nu=1+n_{1}, 2+n_{1}, \cdots, n_{2}$, say; and so on. It is clear that $\omega_{n}=O(1)$, and that $\lim \omega_{n}$ does not exist. Furthermore, for $n \leq n_{1}$, $\omega_{n} \uparrow$, for $n_{1}<n \leq n_{2}, \omega_{n} \downarrow$, and so on. Now

$$
(n+1) \omega_{n+1}-n \omega_{n}=n\left(\omega_{n+1}-\omega_{n}\right)+\omega_{n+1} \geq \frac{3}{2}-1=\frac{1}{2},
$$

hence $n \omega_{n} \uparrow$. Finally

$$
\left|\omega_{m}-\omega_{n}\right| \leq \sum_{\nu=n+1}^{m} \frac{1}{\nu}<\frac{m-n}{n} \rightarrow 0, \quad \text { for } \frac{m}{n} \longrightarrow 1,
$$


hence $\omega_{n}$ is slowly oscillating.

5. Another equivalent result. We first establish the following lemma.

Lemma 3. Suppose that $U_{n} \geq 0$ and increasing, with $U_{0}=0$, and let

$$
\begin{array}{ccc}
b_{n}=\frac{1}{n}\left(U_{n}-U_{n-1}\right), & n \geq 1, & b_{0}=0 ; \\
B_{n}=\sum_{\nu=0}^{n} b_{\nu}, & n \geq 0 .
\end{array}
$$

Then whenever $k=k(n)$ is so chosen that $k / n \rightarrow 0$, as $n \rightarrow \infty$, the two statements

$$
\frac{1}{n}\left(U_{n+k}-U_{n}\right) \rightarrow 0
$$

and

$$
B_{n+k}-B_{n} \rightarrow 0
$$

are equivalent.

Proof. From (5.1) we have

$$
U_{n}=\sum_{\nu=0}^{n} \nu b_{\nu}, \quad U_{n+k}-U_{n}=\sum_{\nu=n+1}^{n+k} \nu b_{\nu}
$$

Now

$$
B_{n+k}-B_{n}=\sum_{\nu=n+1}^{n+k} b_{\nu} \leq \frac{1}{n} \sum_{\nu=n+1}^{n+k} \nu b_{\nu}=\frac{1}{n}\left(U_{n+k}-U_{n}\right)
$$

thus (5.3) implies (5.4). Furthermore,

$$
B_{n+k}-B_{n} \geq \frac{1}{n+k}\left(U_{n+k}-U_{n}\right)
$$

hence (5.4) implies (5.3). This proves the lemma.

We note that 


$$
B_{n}=\frac{1}{n} U_{n}+\sum_{\nu=1}^{n-1} \frac{1}{\nu(\nu+1)} U_{\nu}
$$

and

$$
U_{n}=n B_{n}-\sum_{\nu=0}^{n-1} B_{\nu}
$$

It is an immediate consequence of Lemma 3 that Theorem 3 is equivalent to the following theorem (for a direct proof see [4, Theorem IV]).

THEOREM 4. If

$$
\sum_{\nu=n+1}^{n+k}\left(\left|a_{\nu}\right|-a_{\nu}\right) \rightarrow 0, \quad \text { as } \frac{k}{n} \rightarrow 0, \quad n \rightarrow \infty,
$$

then Abel summability of $\sum_{n=0}^{\infty} a_{n}$ implies convergence of the series to the same value.

A generalization of this theorem to Dirichlet series and to Laplace integrals, on different lines, is given in [3].

\section{REFERENCES}

1. A. Rényi, On a Tauberian theorem of O. Szász, Acta Univ. Szeged. Sect. Sci. Math. 11 (1946), 119-123.

2. O. Szász, Verallgemeinerung eines Littlewoodschen Satzes über Potenzreihen, J. London Math. Soc. 3 (1928), 254-262.

3. - Verallgemeinerung und neuer Beweis einiger Sätze Tauberscher Art, Sitzungsberichte d. Bayer. Akad. d. Wissenchaften $z u$ München, 59 (1929), 325-340.

4. - Generalization of two theorems of Hardy and Littlewood on power series, Duke Math. J., 1 (1935), 105-111.

5. - Introduction to the theory of divergent series, University of Cincinnati, Cincinnati, 1944; Hafner, New York, 1948. 



\title{
EDITORS
}

\author{
HERBERT BUSEMANN \\ University of Southern California \\ Los Angeles 7, California
}

R. M. RoBINSON

University of California

Berkeley 4, California

E. F. BECKENBACH, Managing Editor

University of California

Los Angeles 24, California

ASSOCIATE EDITORS
R. P. DILWORTH
P. R. HALMOS
BQRGE JESSEN
J. J. STOKER
HERBERT FEDERER
HEINZ HOPF
PAUL LÉVY
E. G. STRAUS
MARSHALL HALL
R. D. JAMES
GEORGE PÓLYA
KÔSAKU YOSIDA

\section{SPONSORS}

UNIVERSITY OF BRITISH COLUMBIA

CALIFORNIA INSTITUTE OF TECHNOLOGY UNIVERSITY OF CALIFORNIA, BERKELEY

UNIVERSITY OF CALIFORNIA, DAVIS

UNIVERSITY OF CALIFORNIA, LOS ANGELES

UNIVERSITY OF CALIFORNIA, SANTA BARBARA

OREGON STATE COLLEGE

UNIVERSITY OF OREGON
UNIVERSITY OF SOUTHERN CALIFORNIA STANFORD UNIVERSITY

WASHINGTON STATE COLLEGE

UNIVERSITY OF WASHINGTON

AMERICAN MATHEMATICAL SOCIETY NATIONAL BUREAU OF STANDARDS, INSTITUTE FOR NUMERICAL ANALYSIS

\section{Vari-Type Composition by \\ Cecile Leonard \\ Ruth Stafford}

With the cooperation of

E. F. Beckenbach

E. G. Straus

Printed in the United States of America by

Edwards Brothers, Inc., Ann Arbor, Michigan

UNIVERSITY OF CALIFORNIA PRESS - BERKELEY AND LOS ANGELES COPYRIGHT 1951 BY PACIFIC JOURNAL OF MATHEMATICS 


\section{Pacific Journal of Mathematics}

\section{Vol. 1, No. $1 \quad$ November, 1951}

Ralph Palmer Agnew, Ratio tests for convergence of series............. 1

Richard Arens and James Dugundji, Topologies for function spaces....... 5

B. Arnold, Distributive lattices with a third operation defined ........... 33

R. Bing, Concerning hereditarily indecomposable continua ........... 43

David Dekker, Generalizations of hypergeodesics ............... 53

A. Dvoretzky, A. Wald and J. Wolfowitz, Relations among certain ranges of vector measures.................................... 59

Paul Erdős, F. Herzog and G. Pirani, Schlicht Taylor series whose convergence on the unit circle is uniform but not absolute .......... 75

Whilhelm Fischer, On Dedekind's function $\eta(\tau) \ldots \ldots \ldots \ldots \ldots \ldots . \ldots 3$

Werner Leutert, The heavy sphere supported by a concentrated force ...... 97

Ivan Niven and H. Zuckerman, On the definition of normal numbers ...... 103

L. Paige, Complete mappings of finite groups .................. 111

Otto Szász, On a Tauberian theorem for Abel summability ............. 117

Olga Taussky, Classes of matrices and quadratic fields ............. 127

F. Tricomi and A. Erdélyi, The asymptotic expansion of a ratio of gamma functions .................................... 133

Hassler Whitney, On totally differentiable and smooth functions ......... 143 